\title{
Editorial
}

\section{Research into Anxiety of Childhood: Playing Catch-up (to Olympic Standard)}

This special issue is the culmination of an ESRC seminar series grant awarded to the authors of this editorial. We named the seminar series CATTS (Child Anxiety, Theory and Treatment Seminars) and it took the form of six highly stimulating, one-day seminars on the subject of child anxiety, with participants from clinical and academic backgrounds and from Great Britain, Europe, the USA and Australia. Most of the authors in this publication, and a sister special issue in Cognitions and Emotion (2008), participated in the CATTS series.

One of the main benefits for participants of the CATTS series was increased insight into the enormous range and depth of child anxiety research that is happening around the world. However, as little as 15 years ago, research into childhood anxiety disorders was in its infancy. Few researchers were dedicated specifically to the understanding of these disorders, and fewer still to its treatment. We hope that the papers in this special issue of Behavioural and Cognitive Psychotherapy will leave readers in no doubt that this situation has changed for the better.

In 1994, Philip Kendall published the first randomized controlled trial of an intervention specifically designed for anxiety disorders in youth (Kendall, 1994). A slick of groundbreaking trials followed this pioneering work, but in these early days the work was, by necessity, broadbrushstroke in nature - mapping out tracts of territory roughly and rapidly. Several papers in this issue show that, in a remarkably short space of time, the field has become at once broader and more focused in its remit. Kendall's group (Crawley, Beidas, Benjamin, Martin and Kendall) are once again leading the charge, in this issue presenting research that examines for whom CBT works, and in which circumstances. Their paper explores which socially anxious children benefit most from CBT and, in particular, examines the role of mood in outcomes. Similarly, Marin, Rey, Nichols-Lopez and Silverman examine moderators of anxiety response, with a focus on anxiety sensitivity, and report some interesting gender effects. By contrast, Sze and Wood broaden out the utility of the current treatment approaches. They report a case study showing that CBT can be used effectively, with some thought and modification, to treat anxiety in a child with Autistic Spectrum Disorder.

In a parallel development, other research has devised interventions for child anxiety that have wider applicability and, in particular, that have public health applications. Traditional CBT for childhood anxiety has usually been run with individuals or small groups. This is rather expensive and demands high levels of scarce therapist skills, meaning that it has been available only to the fortunate few. Spence et al. report the development of CBT for youth anxiety that is delivered by computer with minimal therapist contact. They are open about some of the difficulties faced in this endeavour, but present two optimistic case studies of its use. Hardy and Stallard explored the use of symptom monitoring as a simple and cost-effective intervention for children and adolescents who had been involved in a road traffic accident. As Spence et al. point out, interventions that are as minimally demanding on services as these have the potential to be rolled out widely, and even small benefits to clients can translate into large public health payoffs. 
One fruitful approach to the lack of research in the child and adolescent field has been to take well-established ideas from the adult field, and evaluate their applicability for a younger population. Useful strides in both understanding and treating childhood anxiety have been made in this way. Hignett and Cartwright-Hatton examine the role of observer perspective in social anxiety of adolescence, and confirm adult findings on this association. Hodson, McManus, Clark and Doll also examine key aspects of Clark and Wells' (1995) model of social anxiety in young adolescents, confirming that these associate with social anxiety in the same way as they do in the adult literature. Similarly, as mentioned above, two of the papers in this issue confirm that adult-derived interventions (symptom monitoring, CBT) are effective in a younger population. Finally, Reynolds and Reeves reviewed the literature on the applicability of adult models of OCD for children and adolescents. Their conclusions are positive - they could find only one study that did not replicate findings from the adult literature. However, Reynolds and Reeves also point out that it would be unwise to assume that we can downsize all adult models and treatments without pause for thought; the potential for publication biases to mislead this developing field is large.

Meanwhile, other research has taken a bottom-up approach. That is, the research has developed from observations made of children (and their families) and is producing theories and interventions that are not derived from existing protocols for adults. In this issue, PriceEvans and Field studied the impact of parenting style on children's responses to receiving verbal threat information, and report some interesting results, particularly with respect to physiological response to threat. Creswell, O'Connor and Brewin report a novel experimental study of the relationship between parental appraisals of children's coping, and their subsequent parenting behaviour. Finally, Askew, Kessock-Philip and Field report a series of three elegant, experimental studies exploring the interaction between verbal information and vicarious learning in fear acquisition. Again, all of these studies are examples of research that is going beyond demonstrating simple linear or correlational effects. Research is moving towards experimental manipulation of theoretical variables, and examination of complex interactions between these. Although this research is difficult, and frequently produces more surprising or puzzling results (e.g. Askew et al.), it is the way forward.

\section{Acknowledgements}

The authors are grateful to the Economic and Social Research Council (ESRC) for Research Seminar Grant RES-451-25-4103 (Child Anxiety: Developments in Theory and Treatment) without which the seminar series and this publication would not have been possible.

For further details on the CATTS project, the reader is referred to: http://www.statisticshell.com/research/catts.htm

Sam Cartwright-Hatton, Andy Field, Cathy Creswell and Shirley Reynolds Guest Editors

\section{References}

Clark, D. M. and Wells, A. (1995). A cognitive model of social phobia. In R. G. Heimberg and M. R. Liebowitz (Eds.), Social Phobia: diagnosis, assessment, and treatment. New York: Guilford Press.

Kendall, P. C. (1994). Treating anxiety disorders in children: results of a randomized clinical trial. Journal of Clinical Child Psychology, 62, 100-110. 